\title{
Computer-Based Writing Tools and the Cognitive Needs of Novice Writers
}

Robert B. Kozma

B y some estimates, as much as ninety percent of all computer use on the college campus is for word processing (Turner, 1987). Perhaps this is as it should be. Writing is such a ubiquitous and important activity in higher education that if the application of the computer's power can aid the composing process it might make a significant contribution to the quality of college learning. Many common features of word-processing packages make them intuitively appealing. Their ability to easily insert, delete, and replace text would seem to facilitate writing and revision. These capabilities certainly account for the widespread use of word-processing packages as productivity tools in business. While this last fact, by itself, may justify the inclusion of computers in the writing curriculum, it does not address the pedagogical role that computers may play in the improvement of writing.

This article raises several important questions about writing and computers:

- What are the cognitive needs of novice writers? 
- Do word processors provide the kind of help that is particularly useful to those struggling with their writing?

- Are there other ways to use computers to aid the writing process, particularly for novice writers?

This article examines the growing understanding of the cognitive processes of writing and the capabilities (and limitations) of computers and draws implications for the design and use of computer-based tools to improve writing and to conduct research in this area.

\section{Cognitive Processes of Expert and Novice Writers}

Research on the differences between novice and expert writers comes out of the larger body of research on the writing process. Over the past ten years, several researchers (Flower \& Hayes, 1981; Bereiter \& Scardamalia, 1987) have observed writers, as they compose, develop and refine a cognitive model of the writing process. Flower and Hayes characterize the cognitive activities of the writing process as a flexible, recursive movement between planning, translating, and reviewing. While in the planning stage, for example, the writer may translate the plans into text, or review and revise the plans. These processes are also hierarchical; for example, while working on a mid-level goal of "writing a catchy opening," the writer may translate this into the lower-level goal of writing the first sentence, or evaluate this against the higherlevel rhetorical goal of "convincing readers of contrary opinion that my position is reasonable."

The process is constrained by information in long-term memory such as topic-relevant information, knowledge and expectations of audience, and grammatical rules and rhetorical strategies. It is also constrained by limited capacity in short-term memory. For example, using short-term memory for nonautomated skills related to grammar or spelling reduces the space that is available for planning and rhetorical analysis. If capacity is reached, ideas may be lost, goals may be forgotten, and performance will deteriorate.

Despite the robustness of this general model, important differences have emerged in studies that have compared expert writers and novices. Depending on the study, "novices" may be those whose writing is judged to be of poorer quality-children or first-year college students. "Experts" may be those judged to have better compositionsadult writers, writing teachers, professional writers, or advanced college students. All of these writers can be arrayed along a continuum of writing experience. More experienced writers have more knowledge, 
skill, and strategies of the sort mentioned above, and with increased experience these become more automatized and make fewer demands on short-term memory. Differences in experience also result in characteristic patterns of cognitive behavior during writing that distinguish between more and less experienced writers, as described below. Novice writers and the ways computer-writing tools might help them are the focus of this article.

One of the most distinctive characteristics of novice writers is that they oversimplify the representation of the task (Bereiter \& Scardamalia, 1987). While experienced writers may represent an assignment as "establishing the benefits of my position to readers likely to hold the opposing view to mine," novices may see the same task as "writing down my position on this topic." Consequently, novices have few cues to use in effectively searching their long-term memory. This thin representation also gives them few criteria to use in sorting the information they retrieve and as a result almost everything that novices recall appears in the text. This is a pattern Bereiter and Scardamalia (1987) refer to as "knowledge telling." Novices have vague top-level goals and spend more time at lower-level goals, dealing with the surface structure of the text (Flower \& Hayes, 1981). Thus, they are text-bound, reluctant to jump from surface or word levels to more global decisions, such as "What do I want to say?" (Bridwell, 1980; Perl, 1979; Pianko, 1979; Sommers, 1980).

Experts, on the other hand, tend to formulate the task in terms of two, sometimes dissonant, sets of problems and gcals: those related to topical ideas and those related to their expression in the text. The richness of this representation provides more probes for memory search. But more importantly, the dialectic between content issues and rhetorical issues results in the reciprocal translation of intentions into text and text into intentions, a pattern Bereiter and Scardamalia (1987) refer to as "knowledge transformation." As a result, experts generate much more content than will appear in the final product. They also make more sentence- and theme-level changes based on incongruities between their text and their intentions, and their revisions are more likely to change the meaning of the composition.

\section{Computers and Learning}

A crucial goal for composition instructors is to help novice writers move from knowledge tellers to knowledge transformers. So, are there capabilities that computers have that can be used to facilitate this transition? 
Kozma (in press-a) characterizes learning with media, including computers, as a complementary process within which representations are constructed and procedures performed sometimes by the learner and sometimes by the medium. Computers have a unique set of capabilities among educational media. They not only present information in a range of symbol systems, but also they have the ability to receive, store, process, and manage this information. These are capabilities that enable the computer to construct representations and to perform procedures that students may be unable to do for themselves.

But as powerful as computers are, the mere introduction of computers (or any medium) into the classroom does not improve learning (Clark, 1985). Their effectiveness depends on instructional methods, or the ways in which computers' capabilities are used. In higher education, the dominant way computers are used is as a tool. To aid learning, computer-based tools can (Salomon, 1988)

- assume part of the intellectual burden by handling lower-level functions of the task, thus enabling learners to work at higher-levels;

- provide learners with guidance by raising questions, signaling errors, suggesting moves, etc.;

- display intermediate states and processesen route to the final solution; and

- provide models of how information can be represented and processed.

Salomon suggests that at least under certain circumstances these capabilities may become internalized and used by the learner even when the tool is no longer available.

Corno and Snow (1986) contend that the effectiveness of instructional interventions also depends on student abilities: methods that work for some students do not work for others. More structured interventions tend to benefit learners who are new to a domain by assisting them with cognitive processes that they are not yet able to perform for themselves. However, highly structured interventions may interfere with the preferred strategies of more able learners or those more experienced in a particular domain. Experienced learners benefit more from less structured environments because these interventions allow or prompt more able students to draw on their own considerable capabilities and interests. Low ability or novice students are likely to perform worse with these interventions. 
These general considerations have several implications for the role computers-based tools may play in aiding novice writers. First of all, they suggest that software which is effective for experienced writers may not work (indeed, may even be harmful) for those still developing their writing skills. They also suggest that such tools will be more effective if they activate the task-relevant cognitive skills that writers already have while they model and support those cognitive processes and skills the writers lack. This then is the essential tension embedded in the symbiotic relationship between tool and user: are the skills needed to employ the capabilities of the tool already available within the learners, and do the capabilities of the tool provide enough support for learners who are still developing their skills?

\section{Researching and Developing Computer-Based Writing Tools}

There is a growing range of computer-based writing tools that are available to composition instructors. Of particular interest in this article is software with functionality that goes beyond word-processing programs, for these are the tools specifically designed to support learners still developing their expertise.

Certainly word-processing programs help writing by automating some of the more burdensome aspects of text production and revision. Features such as inserting, deleting, and moving blocks of text are likely to account for the findings that students who use word-processing programs write longer compositions, have fewer errors, and make more revisions (Hawisher, 1988, 1989). However, these features have little to do with the formulation of plans, the retrieval and organization of knowledge, or the evaluation of the emerging text: those cognitive processes least prevalent among novice writers. Indeed, Haas (1989) found that writers planned less when they used word-processing programs rather than when they used a pen.

Novice writers, given their cognitive needs, are likely to benefit from features that go beyond those in standard word-processing programs, features that correspond more directly to cognitive components of the composing process. Software designed with functions that model or that prompt the developing cognitive skills of novice writers may be more likely to scaffold their writing processes and improve their written compositions. Novices may benefit most from software that assists in the formulation of rhetorical goals and strategies, aids the 
recall and organization of topical knowledge, assists in the translation of goals and knowledge into text, and prompts the revision of plans as well as text.

The following are various types of software tools that may prove useful to novice writers. Additional discussions of such tools appear in articles by Pea and Kurland (1987), Frase (1987), Rodrigues and Rodrigues (1984), and Schwartz (1984).

\section{Idea Organizers}

These packages prompt the writer to consider the organization, structure, or relationship among the various ideas he or she generates. Some idea organizers provide a work space which by its structure and functionality prompts the user to arrange the sequence and subordination of ideas into a standard outline form. Examples include ACTA and MORE (Living Videotext, Inc.). These outlining functions have begun to appear in standard word-processing programs, such as MICROSOFT WORD. MORE also allows the user to graphically display these hierarchical relationships as a tree structure. Other packages, such as Xerox's NOTECARDs (Xerox Palo Alto Research Center) and LEARNING TOOL (Kozma \& Van Roekel, 1986), use a graphic interface to prompt the consideration of relationships, in addition to those that are hierarchical. Employing a node-and-link structure, these packages prompt the user to label, and thus consider relationships such as causality, temporality, functionality, etc. The result is a network of ideas and relationships that can guide the generation of the text.

Kellogg (1989) points out that tools such as idea organizers reduce the cognitive load of the user by focusing attention on the planning process while temporarily ignoring the translating and reviewing processes. This corresponds to the need of novice writers to spend more time thinking about the higher-level structure of the text and their writing plans. It may counteract the novice writer's tendency to focus immediately on the surface of the text, since to use these tools at all requires the consideration of the structure of the text and relation among ideas. The consideration of relationships may also result in the generation of additional cues that can be used to probe memory.

There is, however, a problematic assumption that underlies this type of software: that the user can convert intermediate plans, as represented by outlines and networks, into written discourse and that these plans can be used subsequently to assess the quality of the emerging text. There is little functionality built into these packages that assists this transformation process. This may be particularly problematic for node-and-link representations that must ultimately be 
converted to linear text. The gap between plans, text, and revision may be a chasm across which only a few novice writers can leap.

\section{Text Analyzers}

These tools have editorial functions that allow the user to review a draft for such things as word frequency, wordiness, readability, and the use of passive voice and nominalizations. They also contain proofreading functions that allow the writer to check spelling, punctuation, capitalization, subject/verb agreement, and split infinitives. WRITR's WORKBENCH and GRAMMATIK are examples of these packages. Text analyzers locate the specific problems and provide the user with a summary report that might contain the number of sentences, the average number of words in a sentence, the frequency of various verb forms, and so on. Some text analyzers include an assessment that compares the summary statistics of the user's text with a norm derived from the research literature and accompanies it with advice on how problems might be corrected.

Keifer and Smith $(1983,1984)$ found that when provided with a passage, college students who used WRITER's WORKBENCH made more changes related to clarity and style than students who did not use the software during their composing course. There were no differences, however, between the groups on holistic scores of essays the students composed at the end of the course.

Text analyzers should be used carefully with novice writers. These packages focus more on the product than the process of writing. They prompt the writer to review the surface features of the text for changes in word or style. While such packages may facilitate editing and proofreading tasks, these are not the problems that plague novice writers most. When revising, novice writers have more difficulty creating an interplay between text and goals such that the emerging text is evaluated against intended outcomes and these intentions in turn are revised as they become expressed in text. A focus on surface changes may reduce the likelihood that deeper changes occur; at any rate, there is nothing about text analyzers that will facilitate this deeper analysis.

\section{Process Prompters}

Process prompters are specifically designed to assist writers with various components of the writing process. These packages have functions and prompts that support the planning, translation, and review processes. Some examples of these are HBJ WRITER, WORDBENCH, and WRTER'S HELPER, STAGE I. 
For example, WRITER'S HELPER, STACE II, winner of the 1988 Educom/ Ncriptal Award for Best Writing Software, has planning activities to help students find topics (Brainstorms, Lists, Questioner, Associations, Starters, Idea Wheel), explore creative approaches to their topics (Crazy Contrasts, Three Ways of Seeing, Audience, Random Revelations, and Connections), and plan and organize their compositions (Trees, Debating an Issue, Goals, Compare and Contrast, and Outliner). Once students have completed planning activities, they can move their notes to the word-processing program and compose their text. Students can use a variety of tools to analyze the emerging structure of the text and review it for usage, diction, readability, etc.

Because of the correspondence between their features and the writing process, these packages are particularly promising for novice writers. Yet, they also highlight the essential tension built into computer-based tools: it is not clear that novices have enough skill to use the capabilities of process prompters or that prompting is a sufficient intervention for novices. In addition, these packages are among the most operationally complicated, thus introducing another layer of complexity on top of the writing task itself.

A study by Kozma (in press-b) illustrates the complex relationship between the skill of the student and the capabilities of computer-based writing tools. He examined the impact (1) of prompts, similar to those used in the packages above, embedded in a word-processing program and (2) of two kinds of idea organizers - an outliner and a graphic, node-and-link organizer. Kosma found that the prompts asked questions related to topic, reader, and goal. Students who received the prompts or either of the idea organizers did more planning than those who did not. Students who received both the prompts and one of the idea organizers did the most planning. However, planning corresponded to better compositions only under certain conditions; there were interactions between treatments and writing experience. Novice writers used the topic prompts to help them generate content, but they had great difficulty using the reader and goal prompts. Novices that had the outliner with prompts used it to help them construct a linear structure for this topical information, and they wrote better compositions. On the other hand, the reader and goal prompts evoked the emerging rhetorical skills of the advanced writers and the graphic idea organizer allowed them to represent and to access this rhetorical information and to write better compositions.

While one set of tools and prompts helped advanced writers use their emerging rhetorical skills to become better knowledge transformers, different tools and prompts helped novice writers become better 
knowledge tellers. The novice writers did not have the skills needed to use the rhetorical prompts and the graphic idea organizer, and this intervention was not powerful enough to help these students acquire skills that they did not yet have.

\section{Communication Packages}

While many computer-based tools are limited in the support they can provide to novice students, communications packages extend this capability by electronically connecting the student to the teacher and other students. Some packages allow the instructor to easily comment on a student's text. This can be done on-line, in a network system using software such as COMMENTS in which the instructor can observe and comment on students' writing in progress. Or, it can be done in an offline manner, using packages such as PROSE in which the instructor can insert comments in an electronic draft which is returned to the student on a disk.

Communication packages can also connect a student with others to form a community of writers. Whether one takes the cognitive orientation of Flower (1979), or the social orientation of Bizzell (1982) or Bruffee (1984), the social context of writing is important. Novice writers have a limited perspective in their writing and are not experienced members of a text-based discourse community. Writing within a social environment can integrate the writer into the community's norms, expectations, and conventions. An internal representation of these expectations can create the dialectic between intentions and text and build knowledge transformation skills.

With this application of communication software, students interact with the teacher and each other in a computer-mediated conferencing environment to discuss assignments, topics, ideas, problems, and drafts (Spitzer, 1989). Ann Hill Duin, winner of the 1989Educom/Ncriptal Best Curriculum Innovation Award in Writing, used COLLABORATIVE WRITING on a network to help the students in her technical writing course collaboratively plan and compose memos, reports, and other nonacademic writing tasks. The software prompts and tutors students through planning, drafting, and revising and facilitates the exchange of drafts and messages in which students can give and receive feedback.

DAFDALus, winner of the 1990 Educom/Ncriptal Award for Best Writing Software, uses similar prompt and mail features supplemented by a real-time conferencing system that enables students and instructors to conduct intensive, text-based class discussions over the computer network. Alternatively, some software, such as SEEN creates a "virtual network" among stand-alone machines by using a message or 
mailbox system that students pass around on disk. These programs provide writers with the reactions of an audience and facilitate the students' articulation, discussion, and analysis of the writing process. This approach may provide novice writers with information, motivation, and feedback that they may not otherwise have.

\section{Artificially Intelligent Writing Environments}

Even the most sophisticated of the current writing packages rely heavily on prompting as their primary pedagogical strategy. Yet, Corno and Snow (1986) point out that less skilled learners are likely to require more instructional support than this. Software packages designed for novices may need to "understand" the difficulty a student is having and to provide advice that specifically addresses this difficulty. This capability is normally associated with artificial intelligence (AI), and there are two ways that $\mathrm{AI}$ is used to develop writing environments: one focuses on the written product, the other focuses on the writing process. Both of these approaches are still in the early development stages.

Kieras (1989) is developing an artificial intelligence (AI) writing system that analyzes a written document and identifies problematic passages. This approach is similar to the text analyzers discussed above except that the assessment is based on the comprehensibility of the text rather than on grammar, punctuation, and other surface features. The system uses an "expert model" of text comprehension (e.g., Kintsch \& Van Dijk, 1978) to analyze the cohesive structure of the text. For example, as it progresses through the text, the system can identify and parse noun phrases and match each new noun phrase against previous references. Poor connections between noun phrases and previous references reduce the comprehensibility of the text and these would be listed in a report to the student. PARNASSUS, provides the students with a deeper semantic analysis of the text. Because computer-based natural language processing is still limited, the system makes deep semantic analysis feasible by restricting the students to a provided text passage rather than allowing them to write on any topic they choose. Students are given a passage, a section is marked as problematic, and their task is to rework the sentences. The system allows them to generate their own "corrected" sentences and provides them with semantic and rhetorical feedback.

Smith and Lansman (1989) are pursuing the second approach to AI writing environments, one that focuses on the process rather than the product. They have built a system, called WE (for WRIIING ENVIRONMENT), that provides the user with features, functions, and work spaces built on 
an expert model of the writing process (Flower \& Hayes, 1981). The program records the writer's use of these work spaces and functions and analyzes these data to make inferences about the user's cognitive processes. In the future, such a system might use this information to guide the writing process. For example, it might analyze the writer's pauses and detect that he or she is having difficulty developing the opening paragraph of the composition. It might also detect a limited use of the planning space to make an inference that the writer has not fully developed the topic or has not referred to information that has been developed. The program might then give the user advice on the need to build, to modify, or to refer to plans and topic-related goals.

\section{Needed Research}

It may only be after advanced AI systems are fully developed that computer-based tools will have the capabilities needed to aid novice writers. But, the development of these ad vanced writing environments relies heavily on research in the areas of composition, text comprehension, and the roles computers play in these. Additional fine-grained, moment-by-moment analyses of the writing process are essential for the development of formal computer models of expert and novice behavior needed to build these systems. In-depth analyses of the component processes of writing, such as that of the translation or text production process by Kaufer, Hayes, and Flower (1986) or the reviewing process by Hayes and Flower, Schriver, Stratman, and Carey (1985), provide the parameters of novice and expert behavior needed by these models to identify dysfunctional behavior and to provide advice.

Research that examines student use of computer-based writing tools is also needed, and this research needs to be more processoriented. While there are many studies of word-processing packages, most look only at "whether it works" and not at "how it is used." And there are few studies that look at writing software other than wordprocessing programs. The design of computer-based writing environments would be advanced by research that looks at which features, functions, or prompts most help which components of the writing process. More information is also needed on the differential effects of these tools on novices and experts. This effort would benefit from research that uses longitudinal designs, focuses on experienced users of computers and examines the interaction of computers and other classroom activities (Hawisher, 1989). 


\section{Conclusions}

The use of word-processing packages has been an important addition to the writing classroom, an addition that has introduced students to tools they will use in their professional careers. Word-processing packages have also helped students to write more and to make more revisions and fewer errors. Beyond word-processing packages, there is a growing menu of tools that are designed to facilitate idea generation, planning, and revision. These can be profitably used to evoke and to support the emerging skills of more advanced writing students. They are likely to be insufficient, by themselves, for the needs of novice writers. Until the development of more powerful AI environments, writing tools are best used with novices as part of a well planned, process-oriented writing curriculum.

There are several ways composition teachers can use these tools to support the development of novice writers. A particular feature of a tool can be introduced in conjunction with instruction on a corresponding component of the process-oriented curriculum. For example, the instructor can use idea outliners to introduce the development of text structure or use process prompters to introduce rhetorical issues and considerations. The instructor can demonstrate and model internal and otherwise unobservable processes, plans, ideas, and thoughts by thinking aloud while using these tools. Similarly, students' use of thinkaloud protocols can make their cognitive processes available for inspection and comment by the instructor or even by other students. The instructor can then provide the support and guidance that is needed by novices but currently unavailable in computer-based tools. While future writing environments may provide students with customized assistance and advice, this role, for the foreseeable future, remains that of the writing teacher.

Robert Kozma teaches of the University of Michigan in Ann Arbor. 


\section{References}

Bereiter, C., \& Scardamalia, M. (1987). The psychology of written composition. Hillsdale, NJ: Erlbaum.

Bizzell, P. (1982). College composition: Initiation into the academic discourse community. Curriculum Inquiry, 12(2), 191-207.

Brid well, L. (1980). Revising strategies in twelfth grade students' transactional writing. Research in the Teaching of English, 14(2), 197-222.

Bruffee, K. (1984). Collaborative learning and the "conversion of mankind." College English, 46, 635-52.

Clark, R. (1985). Confounding in educational computing research. Journal of Educational Computing Research, 1(2), 129-140.

Corno, L., \& Snow, R. (1986). Adapting teaching to individual differences among learners. In M. Wittrock (Ed.), Handbook of research on teaching. New York: Macmillan.

Duin, A., DeBower, M., Jorn, L., \& Johnson, D. (1989). Collaborative wrinng. [Computer software] Stony Brook, NY: Research Design Associates, Inc.

Dunham, D. (1987). ACTA. [Computer Software] Mesa, AZ: Symmetry Corporation.

Flower, L. (1979). Writer-based prose: A cognitive basis for problems in writing. College English, 41, 19-37.

Flower, L., \& Hayes, R. (1981). A cognitive process theory of writing. College Composition and Communication, 32, 365-387.

Frase, L. (1987). Creating intelligent environments for computer use in writing. Contemporary Educational Psychology, 12, 212-221.

Freidman, M., Rand, E., Von Blum, R., Cohen, M., Gerrard, L., Magpantay, A., Cheng, S., Pisano, A., \& Mak, L. (1986). HBJ Writer. New York: Harcourt, Brace, Jovanovich.

Haas, C. (1989). How the writing medium shapes the writing process: Effects of word processing on planning. Research in the Teaching of English, 23, 181207.

Hawisher, G. (1988). Research update: Writing and word processing. Computers and Composition, 5(2), 7-27.

Hawisher, G. (1989). Research and recommendations for computers and composition. In G. Hawisher and C. Selfe (Eds.), Critical perspectives on computers and composition instruction (pp. 44-73). New York: Teachers College Press.

Hayes J., Flower, L., Schriver, K., Stratman, J., \& Carey, L. (1985). Cognitite processes in revision. Pittsburgh, P'A: Communications Design Center, Carnegie Mellon University.

Kaufer, D., Hayes, J., \& Flower, L. (1986). Composing written sentences. Research in the Teaching of English, 20<2), 121-141. 
Keifer, K., \& Smith, C. (1983). Textual analysis with computers: Tests of Bell Laboratories' computer software. Research in the Teaching of English, 17(3), 201-214.

Keifer, K., \& Smith, C. (1984). Improving students' revising and editing: The Writer's Workbench System. In W. Wresch (Ed.), The computer in composition instruction. Urbana, IL: NCTE.

Kellogg, R. (1989). Idea processors: Computer aids for planning and composing text. In B. Britton and S. Glynn (Eds.), Computer writing environments: Theory, research, and design (pp. 57-87). Hillsdale, NJ: Lawrence Erlbaum Associates.

Kieras, D. (1989). An advanced computerized aid for the writing of comprehensible technical documents. In B. Britton and S. Glynn (Eds.), Computer writing environments: Theory, research, and design (pp. 143-165) Hillsdale, NJ: Lawrence Erlbaum Associates.

Kintsch, W., \& Van Dijk, T. (1978). Toward a model of text comprehension and production. Psychological Review, 85, 363-394.

Kozma, R. (in press-a). Learning with media. Review of Educational Research.

Kozma, R. (in press-b). The impact of computer-based tools and embedded prompts on writing processes and products of novice and advanced college writers. Cognition and Instruction.

Kozma, R., \& Van Roekel, J. (1986). LEARNING TOOL [Computer software] Santa Barbara, CA: Intellimation.

Kurland, D., Levine, J., Olson, C., Ruopp, R., Smith, F., \& Wheeler, F. (1988). WORDBENCH[Computer Software] New York: Addison-Wesley.

Neuwirth, C. (1988). CommENTs [Computer Software] Pittsburg, PA: ICECware.

Neuwirth, C. (1989). Intelligent tutoring systems: Exploring issues in learning and teaching writing. Computers and the Humanities, 23, 45-57.

Pea, R., \& Kurland, D. (1987). Cognitive technologies for writing. In E. Rothkopf (Ed.), Review of Research in Education (Vol. 14), Washington, DC: American Educational Research Association.

Perl, S. (1979). The composing processes of unskilled college writers. Research in the Teaching of English, 13, 317-336.

Pianko, S. (1979). A description of the composing processes of college freshman writers. Research in the Teaching of English, 13(1), 5-22.

Rodrigues, R., \& Rodrigues, D. (1984). Computer-based invention: Its place and potential. College Composition and Communication, 35(1), 78-87.

Salomon, G. (1988). Al in reverse: Computer tools that turn cognitive. Journal of Educational Computing Research, 4, 123-33.

Schwartz, H. (1984). Teaching writing with computer aids. College English, 46(3), 239-247.

Schartz, H. (1988). SEEN [Computer Software] lowa City, IA: Conduit. 
Smith, J. and Lansman, M. (1989). A cognitive basis for a computer writing environment. In B. Britton and S. Glynn (Eds.), Computer writing environments: Theory, research, and design. Hillsdale, NJ: Lawrence Erlbaum Associates.

Sommers, N. (1980). Revision strategies of student writers and experienced adult writers. College Composition and Communication, 31, 378-388.

Spitzer, M. (1989). Computer conferencing: An emerging technology. In G. Hawisher and C. Selfe (Eds.), Critical perspectives on computers and composition instruction (pp. 187-200). New York: Teachers College Press.

Taylor, P., Butler, W., Carter, L., \&Kemp, F. (1990). DaEDalus INSTRUCTONAL SYSTEM [Computer software] Austin, TX: The Daedalus Group, Inc.

Turner, J. (1987). Drive to require students to buy computers slows. The Chronicle of Higher Education, \#33(21), 1, 28.

Wresch, W. (1988). WRTIER's HELPER: STACE II [Computer Software] Iowa City, IA: Conduit. 